\title{
Epilepsia
}

\section{Four-dimensional functional cortical maps of visual and auditory language: Intracranial recording}

\author{
Yasuo Nakai $^{1,2}$ | Ayaka Sugiura $^{1}$ | Erik C. Brown ${ }^{3}$ | Masaki Sonoda ${ }^{1}$ | Jeong-Won Jeong ${ }^{1,4}$ | \\ Robert Rothermel $^{5}$ | Aimee F. Luat ${ }^{1,4}$ | Sandeep Sood ${ }^{6}$ | Eishi Asano ${ }^{1,4}$ (if
}

${ }^{1}$ Department of Pediatrics, Detroit

Medical Center, Children's Hospital of

Michigan, Wayne State University,

Detroit, Michigan

${ }^{2}$ Department of Neurological Surgery,

Wakayama Medical University,

Wakayama-shi, Japan

${ }^{3}$ Department of Neurological Surgery,

Oregon Health and Science University,

Portland, Oregon

${ }^{4}$ Department of Neurology, Detroit

Medical Center, Children's Hospital of

Michigan, Wayne State University,

Detroit, Michigan

${ }^{5}$ Department of Psychiatry, Detroit Medical Center, Children's Hospital of

Michigan, Wayne State University,

Detroit, Michigan

${ }^{6}$ Department of Neurosurgery, Detroit Medical Center, Children's Hospital of Michigan, Wayne State University,

Detroit, Michigan

\section{Correspondence}

Eishi Asano, Department of

Neurodiagnostics, Children's Hospital of

Michigan, Wayne State University,

Detroit, MI.

Email: easano@med.wayne.edu

Funding information

National Institutes of Health, Grant/Award

Number: MH107512, NS047550,

NS064033, NS089659

\section{Summary}

Objective: The strength of presurgical language mapping using electrocorticography $(\mathrm{ECoG})$ is its outstanding signal fidelity and temporal resolution, but the weakness includes limited spatial sampling at an individual patient level. By averaging namingrelated high-gamma activity at nonepileptic regions across a large number of patients, we provided the functional cortical atlases animating the neural dynamics supporting visual-object and auditory-description naming at the whole brain level.

Methods: We studied 79 patients who underwent extraoperative ECoG recording as epilepsy presurgical evaluation, and generated time-frequency plots and animation videos delineating the dynamics of naming-related high-gamma activity at 70-110 Hz.

Results: Naming task performance elicited high-gamma augmentation in domain-specific lower-order sensory areas and inferior-precentral gyri immediately after stimulus onset. High-gamma augmentation subsequently involved widespread neocortical networks with left hemisphere dominance. Left posterior temporal high-gamma augmentation at several hundred milliseconds before response onset exhibited a double dissociation; picture naming elicited high-gamma augmentation preferentially in regions medial to the inferior-temporal gyrus, whereas auditory naming elicited high-gamma augmentation more laterally. The left lateral prefrontal regions including Broca's area initially exhibited high-gamma suppression subsequently followed by high-gamma augmentation at several hundred milliseconds before response onset during both naming tasks. Early high-gamma suppression within Broca's area was more intense during picture compared to auditory naming. Subsequent lateral-prefrontal high-gamma augmentation was more intense during auditory compared to picture naming.

Significance: This study revealed contrasting characteristics in the spatiotemporal dynamics of naming-related neural modulations between tasks. The dynamic atlases of visual and auditory language might be useful for planning of epilepsy surgery. Differential neural activation well explains some of the previously reported observations of domain-specific language impairments following resective epilepsy surgery. Video materials might be beneficial for the education of lay people about how the brain functions differentially during visual and auditory naming.

\section{K E Y W O R D S}

event-related high-gamma modulation, four-dimensional (4D), intracranial electroencephalography (iEEG) recording, physiological high-frequency oscillations (HFOs), speech 


\section{1 | INTRODUCTION}

You might say "cat," "kitty," or "kitten," if you witness one walking in the street. You might answer "bird" or "plane," if you are verbally asked: "What flies in the sky?" Word finding abilities in the visual or auditory domain are crucial for humans. Visual object naming (referred to as "picture naming" below) and auditory description naming ("auditory naming") are commonly used for evaluation of baseline language competence as well as for localization of language-related eloquent areas in patients scheduled to undergo epilepsy surgery. ${ }^{1}$ Transient language impairment elicited by electrical stimulation and task-related neural activation on electrocorticography (ECoG) or functional MRI (fMRI) are useful biomarkers for localization of eloquent areas in clinical practice. ${ }^{2-4}$ The strength of ECoGover fMRI-based language mapping is its outstanding signal fidelity and temporal resolution. ${ }^{5}$ Augmentation of high-gamma ECoG activity is an excellent summary measure of cortical activation, since it is tightly associated with increased neuronal firing rate, ${ }^{6}$ hemodynamic activation, ${ }^{7}$ glucose metabolism, ${ }^{8}$ probability of stimulation-induced language impairment, ${ }^{9}$ and risk of postresection language impairment. ${ }^{10,11}$ In contrast, it remains less certain if taskrelated attenuation of alpha/beta activities is linearly correlated with the underlying cortical activation. ${ }^{12,13}$ Although ECoG can sample only selected regions for each patient, averaging of task-related high-gamma activity ${ }_{70-110 \mathrm{~Hz}}$ at nonepileptic regions across a large number of patients has effectively yielded a movie animating the dynamics of auditory naming-related neural modulation at the wholebrain level. ${ }^{12}$ In this study of 79 patients with focal epilepsy (Table 1), we evaluated the spatiotemporal dynamics of picture and auditory naming-related high-gamma activity at 7348 electrode sites across 66 regions of interest (ROIs). We believe the resulting dynamic atlases of visual and auditory language would be useful for planning epilepsy surgery. Previous behavioral studies of patients undergoing epilepsy surgery indicated that focal resection of a region outside the lower-order sensory areas (ie, primary visual/auditory areas in the present study) may impair naming function of a certain domain but preserve others. ${ }^{14}$ Therefore, we hypothesized that domain-specific namingrelated high-gamma modulation would be evident not only in the primary visual/auditory areas but also in association areas. We expected that this study would clarify the timing of given naming-related neural modulations including activation and suppression at an order of tens of milliseconds, whereas our previous studies of smaller samples focused on neural activation alone at limited numbers of ROIs. ${ }^{11,15}$ Evaluation of task-related neural suppression is important as well, based on the theory of antagonism, suggesting that

\section{Key Points}

- We provided cortical atlases that animate the neural dynamics supporting visual and auditory language function at the whole brain level

- Visual and auditory naming tasks elicited early high-gamma augmentation in domain-specific sensory areas respectively

- Visual and auditory naming tasks commonly elicited early high-gamma augmentation in inferiorprecentral gyri

- Visual and auditory naming tasks elicited domain-specific high-gamma modulations in the left posterior temporal areas

- Visual naming task, compared to auditory, elicited smaller high-gamma augmentation in the left prefrontal areas

effective task completion is facilitated by transient suppression of undesirable cortical function at given moments of performance. $^{16,17}$

\section{2 | METHODS}

\section{1 | Patients}

The inclusion criteria consisted of the following: (a) extraoperative ECoG recording at Children's Hospital of Michigan or Harper University Hospital in Detroit between January 2008 and July 2016, (b) age of 4 years and older, and (c) brain mapping with measurement of naming-related high-gamma activity in visual and auditory domains (Figure 1). The exclusion criteria consisted of the following: (a) brain malformations confounding the anatomic landmarks for the central, calcarine, or lateral sulcus; (b) severe cognitive dysfunction (anticipation of inability to complete naming tasks or verbal intelligent quotient (IQ) $<70$ when available); (c) inability to complete either naming task; (d) primary spoken language other than English; (e) previous epilepsy surgery; (f) visual field deficit on confrontation; and (g) right-hemispheric language dominance as suggested by either Wada test or left-handedness associated with lefthemispheric congenital neocortical lesions. ${ }^{11} \mathrm{We}$ determined handedness based on the observation of preferred hand for writing tasks. No formal testing of handedness (eg, Edinburgh handedness inventory) was performed in this study. The present study was approved by the Wayne State University Institutional Review Board, and written informed consent was obtained from the patients or guardians of patients. 
TABLE 1 Patient profile

\begin{tabular}{|c|c|}
\hline Mean age $(y)$ & 15.2 \\
\hline Median age (y) & 14 \\
\hline Range of age (y) & $5-44$ \\
\hline Range of age of epilepsy onset (y) & $0-34.8$ \\
\hline Proportion of patients with history of GTCS (\%) & 57.0 \\
\hline Proportion of male patients (\%) & 53.2 \\
\hline Proportion of left handedness (\%) & 10.1 \\
\hline \multicolumn{2}{|l|}{ Proportion of sampled hemisphere (\%) } \\
\hline Left & $33(41.8)$ \\
\hline Right & $28(35.4)$ \\
\hline Both & $18(22.8)$ \\
\hline \multicolumn{2}{|l|}{ Seizure-onset zone, ${ }^{\mathrm{a}}$ number of patients (\%) } \\
\hline Left frontal & $11(13.9)$ \\
\hline Left temporal & $26(32.9)$ \\
\hline Left parietal & $8(10.1)$ \\
\hline Left lateral-occipital & $1(1.3)$ \\
\hline Right frontal & $11(13.9)$ \\
\hline Right temporal & $19(24.1)$ \\
\hline Right parietal & $14(17.7)$ \\
\hline Right lateral-occipital & $4(5.1)$ \\
\hline Mean number of antiepileptic drugs & 1.9 \\
\hline Median number of antiepileptic drugs & 2 \\
\hline Range of number of antiepileptic drugs & $1-4$ \\
\hline \multicolumn{2}{|l|}{ Antiepileptic drugs, ${ }^{\mathrm{b}}$ number of patients (\%) } \\
\hline Carbamazepine & $6(7.6)$ \\
\hline Oxcarbazepine & $33(41.8)$ \\
\hline Lacosamide & $26(32.9)$ \\
\hline Zonisamide & $5(6.3)$ \\
\hline Phenytoin & $6(7.6)$ \\
\hline Lamotrigine & $20(25.3)$ \\
\hline Levetiracetam & $32(40.5)$ \\
\hline Valproate & $10(12.7)$ \\
\hline Clobazam & $7(8.9)$ \\
\hline Topiramate & $4(5.1)$ \\
\hline \multicolumn{2}{|l|}{ Etiology, number of patients (\%) } \\
\hline Tumor & $16(20.3)$ \\
\hline Dysplasia & $22(27.8)$ \\
\hline Hippocampal sclerosis & $8(10.1)$ \\
\hline Dysplasia + hippocampal sclerosis & $1(1.3)$ \\
\hline No definitive lesion other than gliosis & $31(39.2)$ \\
\hline Arteriovenous malformation & $1(1.3)$ \\
\hline Mean response accuracy in auditory/picture naming tasks (\%) & $91.3 / 98.1$ \\
\hline
\end{tabular}

Eighty-four percent of patients were 19 years old or younger. Response accuracy was defined as the number of correct trials divided by the number of all trials.

${ }^{\mathrm{a}}$ Sixteen patients had a seizure-onset zone involving at least 2 lobes.

${ }^{\mathrm{b}}$ Fifty-five patients took at least 2 antiepileptic drugs.

\subsection{Acquisition of ECoG and three-dimensional surface MR images}

The detailed methods were described previously. ${ }^{12,18}$ Chronically implanted subdural electrodes $(10 \mathrm{~mm}$ centerto-center distance) recorded ECoG signals at a sampling frequency of $1000 \mathrm{~Hz}$. Preoperative 3T MRI, including fluid-attenuated inversion recovery (FLAIR) images, was visually assessed by an experienced pediatric neuroradiologist who was blinded to scalp video-electroencephalography (EEG) data. Electrode sites classified as seizure-onset zone (SOZ) or those affected by any structural lesion were excluded from further analysis. Sites showing interictal spikes or artifacts during either task were also excluded from analysis. In general, we do not treat sharply contoured waves as interictal epileptiform discharges, if they occur only rarely during the entire recording or they can be attributed to some forms of physiologic waves such as background fluctuations, lambda waves, mu rhythm, and sleep spindles. ${ }^{19,20}$ Thus, a total of 7348 electrode sites were available for further analysis.

Using preoperative volumetric MR image, a threedimensional (3D) surface image was created with the location of electrodes defined on the surface. ${ }^{12}$ The spatial normalization of electrode sites was performed using FreeSurfer scripts (http://surfer.nmr.mgh.harvard.edu). All electrode sites were registered on the averaged FreeSurfer surface image. Each recording site was assigned an anatomic label based on the Desikan-Killiany atlas ${ }^{21}$ (Figure 2). The number of electrode sites eligible for analysis is indicated in Table 2. The statistical power to determine the significance in the degree of high-gamma modulation was dependent on the number of electrode sites at each ROI. For example, the number of electrode sites was 345 and 39 in the left inferior precentral and posterior cingulate regions, respectively; thus, the former ROI had 3.0 times greater statistical power than the latter. Lack of significant high-gamma modulation should be interpreted as failure to demonstrate significant modulation at ROIs where small numbers of electrode sites were available.

\section{3 | Picture and auditory naming tasks}

During the picture naming task, ${ }^{15}$ patients were instructed to overtly name an object presented on an LCD monitor (Experiment Center; SensoMotoric Instruments). Stimuli consisted of common grayscale line-drawn objects (up to 60 objects, such as "cat" and "chair"). ${ }^{22}$ The timings of stimulus presentations, patient behaviors, and ECoG signals were synchronized using a photosensor and microphone. ${ }^{18}$ ECoG traces were aligned to "stimulus onset" and "response onset" (Figure 1A). Trials not accompanied by overt correct answers were excluded from analysis. For 


\section{A Picture naming task}

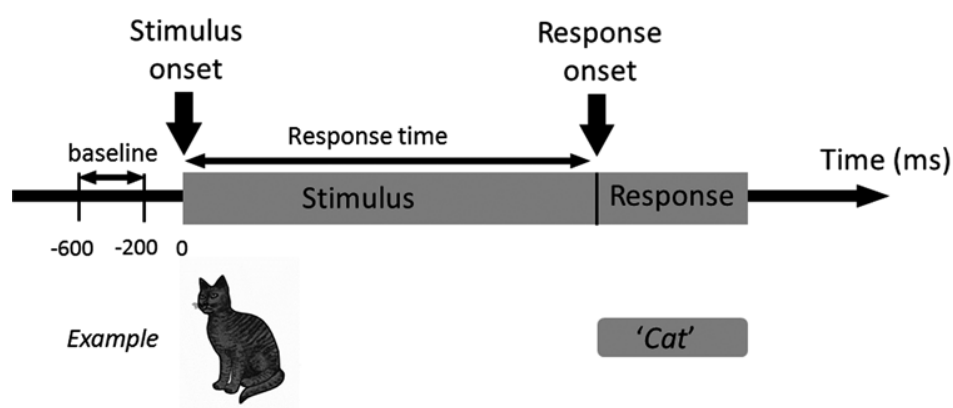

\section{B Auditory naming task}

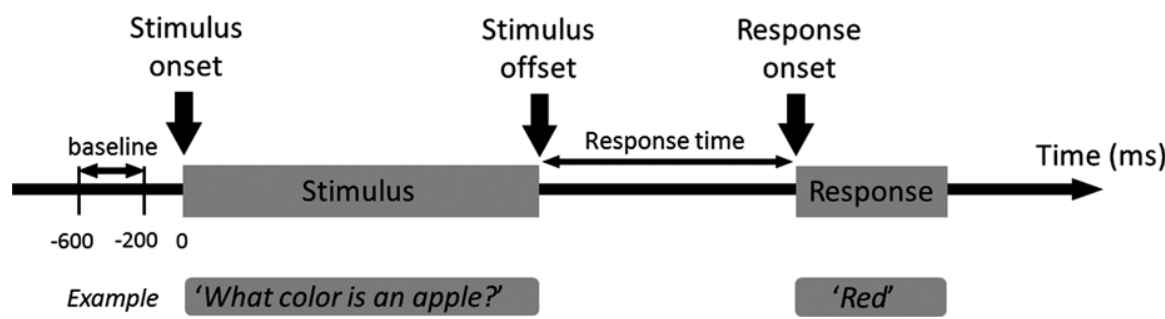

F I G URE 1 Picture- and auditory-naming tasks. A, Picture-naming task: Patients were asked to look at and overtly name each object during extraoperative ECoG recording. At the end of each overt response, the examiner pressed the button to present the next stimulus following a cross in the center of the screen presented for 2 or $2.5 \mathrm{~s}$. We then measured the percent change of high-gamma activity compared to that during the baseline period. The response time was defined as the period between stimulus onset and response onset. B, Auditory naming task: Patients were asked to listen to a series of questions and to overtly verbalize a relevant answer for each question. The median duration across each question was $1.8 \mathrm{~s}$. At the end of each overt response, the examiner pressed the button to present the next stimulus following a silence of 2 or $2.5 \mathrm{~s}$. The response time was defined as the period between stimulus offset and response onset

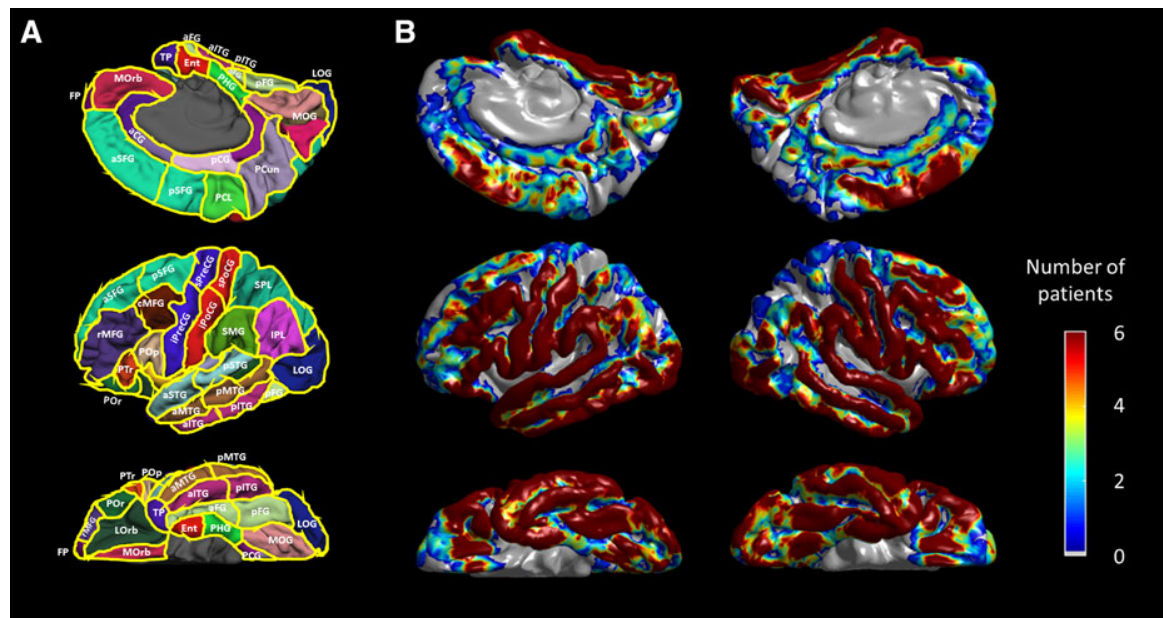

F IG URE 2 Regions of interest (ROIs) and distribution of subdural electrodes included in the analysis. A, aCG, anterior cingulate gyrus; aFG, anterior fusiform gyrus; aITG, anterior inferior temporal gyrus; aMTG, anterior middle temporal gyrus; aSFG, anterior superior frontal gyrus; aSTG, anterior superior temporal gyrus; cMFG, caudal middle frontal gyrus; Ent, entorhinal gyrus; FP, frontal pole; IPL, inferior parietal lobule; iPoCG, inferior postcentral gyrus; iPreCG, inferior precentral gyrus; LG, lingual gyrus; LOG, lateral occipital gyrus; LOrb, lateral orbitofrontal gyrus; MOG, medial occipital gyrus; MOrb, medial orbitofrontal gyrus; pCG, posterior cingulate gyrus; PCL, paracentral lobule; PCun, precuneus gyrus; pFG, posterior fusiform gyrus; PHG, parahippocampal gyrus; pITG, posterior inferior temporal gyrus; pMTG, posterior middle temporal gyrus; POp/PTr/POr, pars opercularis/pars triangularis/pars orbitalis within the inferior frontal gyrus; pSFG, posterior superior frontal gyrus; pSTG, posterior superior temporal gyrus; rMFG, rostral middle frontal gyrus; SMG, supramarginal gyrus; SPL, superior parietal lobule; sPoCG superior postcentral gyrus; sPreCG, superior precentral gyrus; TP, temporal pole. B, The numbers of patients whose ECoG data at a given site contributed to further analysis are presented 
TABLE 2 The number of electrode sites at regions of interest (ROIs)

\begin{tabular}{|c|c|c|}
\hline ROIs & Left & Right \\
\hline aSFG: anterior superior-frontal gyrus & 80 & 118 \\
\hline pSFG: posterior superior-frontal gyrus & 69 & 95 \\
\hline rMFG: rostral middle-frontal gyrus & 249 & 302 \\
\hline cMFG: caudal middle-frontal gyrus & 200 & 175 \\
\hline POr: pars orbitalis of the inferior-frontal gyrus & 90 & 77 \\
\hline PTr: pars triangularis of the inferior-frontal gyrus & 100 & 137 \\
\hline POp: pars opercularis of the inferior-frontal gyrus & 138 & 90 \\
\hline FP: frontal pole & 1 & 8 \\
\hline MOrb: medial orbitofrontal gyrus & 21 & 26 \\
\hline LOrb: lateral orbitofrontal gyrus & 120 & 92 \\
\hline PCL: paracentral gyrus & 29 & 44 \\
\hline sPreCG: superior precentral gyrus & 52 & 64 \\
\hline iPreCG: inferior precentral gyrus & 345 & 296 \\
\hline sPoCG: superior postcentral gyrus & 73 & 44 \\
\hline iPoCG: inferior postcentral gyrus & 270 & 242 \\
\hline PCun: precuneus & 50 & 49 \\
\hline SPL: superior parietal lobule & 46 & 22 \\
\hline SMG: supramarginal gyrus & 291 & 226 \\
\hline IPL: inferior parietal lobule & 84 & 116 \\
\hline aSTG: anterior superior-temporal gyrus & 149 & 134 \\
\hline pSTG: posterior superior-temporal gyrus & 190 & 135 \\
\hline aMTG: anterior middle-temporal gyrus & 111 & 86 \\
\hline pMTG: posterior middle-temporal gyrus & 183 & 87 \\
\hline aITG: anterior inferior-temporal gyrus & 103 & 91 \\
\hline pITG: posterior inferior-temporal gyrus & 114 & 58 \\
\hline aFG: anterior fusiform gyrus & 85 & 102 \\
\hline pFG: posterior fusiform gyrus & 139 & 75 \\
\hline TP: temporal pole & 39 & 50 \\
\hline Ent: entorhinal gyrus & 43 & 64 \\
\hline PHG: parahippocampal gyrus & 22 & 20 \\
\hline LOG: lateral occipital gyrus & 207 & 153 \\
\hline MOG: medial occipital gyrus & 127 & 137 \\
\hline aCG: anterior cingulate gyrus & 14 & 19 \\
\hline pCG: posterior cingulate gyrus & 39 & 41 \\
\hline
\end{tabular}

example, when patients were shown a picture of skunk, incorrect answers would include: "I don't know," "What is this?" or a bad smell gesture. Conversely, correct answers would include "skunk," "squirrel," and so on. A grayscale picture of skunk could be perceived as a squirrel for some individuals. Nouns relevant based on each patient's criteria were treated as correct answers.

During the auditory naming task, ${ }^{12}$ patients were instructed to overtly verbalize an answer for a series of auditory sentence questions (such as "What flies in the sky?"). Up to 100 questions were presented with Presentation (Neurobehavioral Systems). ECoG traces were then aligned to "stimulus onset," "stimulus offset," and "response onset" (Figure 1B). Likewise, trials not accompanied by overt, correct, noun answers were excluded from analysis.

\section{4 | Time-frequency ECoG analysis}

We determined the spatiotemporal dynamics of namingrelated high-gamma modulation using the method identical to that reported previously. ${ }^{12}$ ECoG time-voltage signals were transformed into the time-frequency domain, in steps of $10 \mathrm{msec}$ and $5 \mathrm{~Hz}$, using a complex-demodulation time-frequency transformation. ${ }^{23,24}$ At each 10-msec epoch at each recording site, we measured the percent change in highgamma $_{70-110 \mathrm{~Hz}}$ amplitude (a measure proportional to the square root of power) relative to the mean amplitude during a resting period between 600 and $200 \mathrm{msec}$ before stimulus onset (Figure 1). Time-frequency analysis was repeated with a time-lock to "stimulus onset" and "response onset" for picture naming, whereas "stimulus onset," "stimulus offset," and "response onset" for auditory naming. We plotted the mean and standard error (SE) of high-gamma amplitude change across all available channels at each ROI as a function of time. We determined the timing of statistically significant augmentation (or suppression) of high-gamma activity at each ROI in each hemisphere during each naming task, using studentized bootstrap statistics followed by correction for multiple comparisons across time windows. ${ }^{12}$ Specifically, if high-gamma amplitude was beyond or below the $99.99 \%$ confidence interval (ie, mean $\pm 3.89 \times$ standard error) of that during the resting period, the change in highgamma amplitude was treated as significant at a given ROI commonly in picture and auditory naming tasks. The aforementioned procedures effectively determined at what ROIs common and differential high-gamma modulations were noted during picture and auditory naming tasks. It should be noted that there was no statistically significant difference between "the mean across all electrode sites within each ROI" and "the grand-mean across individual means within each ROI," as shown in Figure S1; therefore, the risk of a single patient's data severely deviating the calculated mean from the true mean was small in the present study. This analytic approach benefited from a maintained statistical power.

High-gamma amplitude measures were spatially presented with a Gaussian half-width at half maximum of $7.5 \mathrm{~mm}$, and sequentially animated on the average FreeSurfer pial surface image as a function of time. Grand-averaging of data from all available patients finally yielded the maps showing the dynamics of picture and auditory naming-related high-gamma modulations at the whole-brain level (Videos S1 and S2). 


\section{3 | RESULTS}

\section{1 | Behavioral results}

We studied 79 patients who satisfied both inclusion and exclusion criteria (Table 1). The mean number of included trials was 55.3 per patient (SE 0.7) during the picture-naming task and 83.2 per patient (SE 2.3) during the auditory naming task. Thus, time-frequency analysis of high-gamma activity during the auditory naming task benefited from an approximately 1.2 times greater signal-to-noise ratio, compared to that during picture naming, because the number of trials was larger during the auditory naming task. The mean response time was $1481 \mathrm{msec}$ (SE $63 \mathrm{msec}$ ) during picture naming and $1407 \mathrm{msec}$ (SE $65 \mathrm{msec}$ ) during auditory naming. Younger age was associated with a marginally longer response time in picture naming $(P=0.07$ on linear regression analysis; regression coefficient $-15,95 \%$ confidence interval $[\mathrm{CI}]-31$ to +1$)$ and auditory naming $(P=0.02$; regression coefficient $-19,95 \% \mathrm{CI}-35$ to -3 ).

\subsection{High-gamma modulation during picture naming}

Video S1 best visualizes the dynamics of naming-related high-gamma modulations in both visual and auditory domains and at the whole brain level. Figures 3 and 4 present snapshots of picture and auditory naming-related high-gamma modulations. Sustaining high-gamma augmentation took place in the bilateral occipital lobes within $100 \mathrm{msec}$ after stimulus onset (Figure 5A,B). High-gamma augmentation subsequently involved the bilateral ventral occipitotemporal pathways (Figure 5E,F) as well as inferior-precentral regions (Figure 5D) at 100-300 msec after stimulus onset. Conversely, high-gamma suppression took place in the left inferior-frontal gyrus including pars orbitalis, triangularis, and opercularis at $300-600 \mathrm{msec}$ after stimulus onset (Figure 5H-J). At $600 \mathrm{msec}$ before response onset and after, high-gamma augmentation involved the pars opercularis of the left inferior-frontal gyrus (Figure $5 \mathrm{H})$. Before and during the response phase, highgamma augmentation involved the bilateral posterior superior-frontal region as well as pre- and postcentral gyri (Figures 3D and 5D). High-gamma augmentation of the left superior-temporal gyrus was minimal before response onset and evident only at response onset and after (Figure 5C).

\section{3 | High-gamma modulation during auditory naming}

Sustained high-gamma augmentation took place in the bilateral superior-temporal gyri within $100 \mathrm{msec}$ after stimulus onset (Figure 5C). High-gamma augmentation subsequently involved the inferior-precentral regions at $100-200 \mathrm{msec}$ after stimulus onset (Figure 5D). Conversely, high-gamma suppression took place in the pars orbitalis of the left inferior-frontal gyrus at 300-600 msec after stimulus onset (Figure $5 \mathrm{H}-\mathrm{J})$. Between stimulus offset and response onset, high-gamma augmentation involved widespread regions of the left hemisphere, whereas high-gamma suppression involved portions of the right lateral prefrontal regions (Figure $4 \mathrm{~B}, \mathrm{C})$. Before and during the response phase, highgamma augmentation involved the bilateral posterior superior-frontal regions as well as pre- and postcentral gyri (Figures 4D and 5D). High-gamma augmentation of the left superior-temporal gyrus was minimal within $300 \mathrm{msec}$ before response onset and evident at response onset and after (Figure 5C).

\section{4 | Spatial-temporal characteristics of domain-specific high-gamma modulation}

Domain-specific high-gamma augmentation was most evident in the primary visual/auditory areas. Specifically, picture and auditory naming tasks elicited high-gamma augmentation in the bilateral occipital and superior-temporal regions within $100 \mathrm{msec}$, respectively (Figure 5A-C). The posterior temporal association neocortex showed domain-specific high-gamma augmentation. Picture-naming task resulted in a medial-to-lateral gradient in the magnitude of high-gamma augmentation at 200-800 msec before response onset, which was greater in the posterior-fusiform and smaller in the posterior middle-temporal region (Figure 5E-G). Conversely, the auditory naming task resulted in a lateral-to-medial gradient in the magnitude of highgamma augmentation in the same time window.

Picture naming, compared to auditory naming, was associated with a smaller high-gamma activity in the left lateral prefrontal regions including the inferior-frontal gyrus (Figure 5H-K). The degree of high-gamma suppression at 328 electrode sites within the left inferior-frontal gyrus at $300-600 \mathrm{msec}$ following stimulus onset was 2.2 times more intense during picture compared to auditory naming $(P=0.01$ on studentized bootstrapping test; -0.029 [ie, $-2.9 \%$ ] during picture naming vs -0.013 [-1.3\%] during auditory naming; Figure 5H-J). Conversely, the degree of high-gamma suppression at 304 right inferior-frontal electrode sites in the same time window was not different between tasks ( $P=0.97 ;-1.0 \%$ during picture naming vs $-0.9 \%$ during auditory naming).

During both naming tasks, lateral prefrontal regions showed high-gamma augmentation with left dominance at 200-800 msec before response onset (Video S1). Such lefthemispheric dominant high-gamma augmentation was more intense in amplitude and more extensive in space and time during auditory compared to picture naming. Auditory 


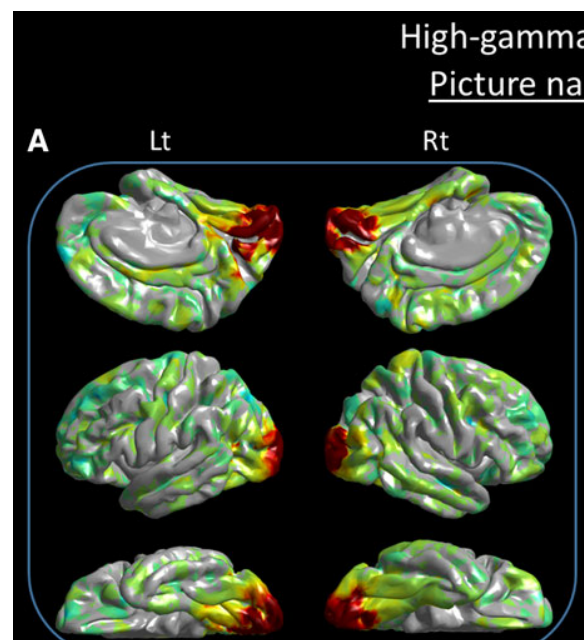

FIGURE 3 Snapshots of picture naming-related high-gamma activity. Event-related amplitude augmentation is reflected by red, whereas suppression is reflected by blue; " +0.4 " indicates that the amplitude was augmented by $40 \%$ compared to the mean during the resting period between -600 and $-200 \mathrm{msec}$ relative to stimulus onset. A, $100 \mathrm{msec}$ after stimulus onset. B, $300 \mathrm{msec}$ after stimulus onset. C, $400 \mathrm{msec}$ prior to response onset. D, Response onset
$100 \mathrm{~ms}$ after stimulus onset

c
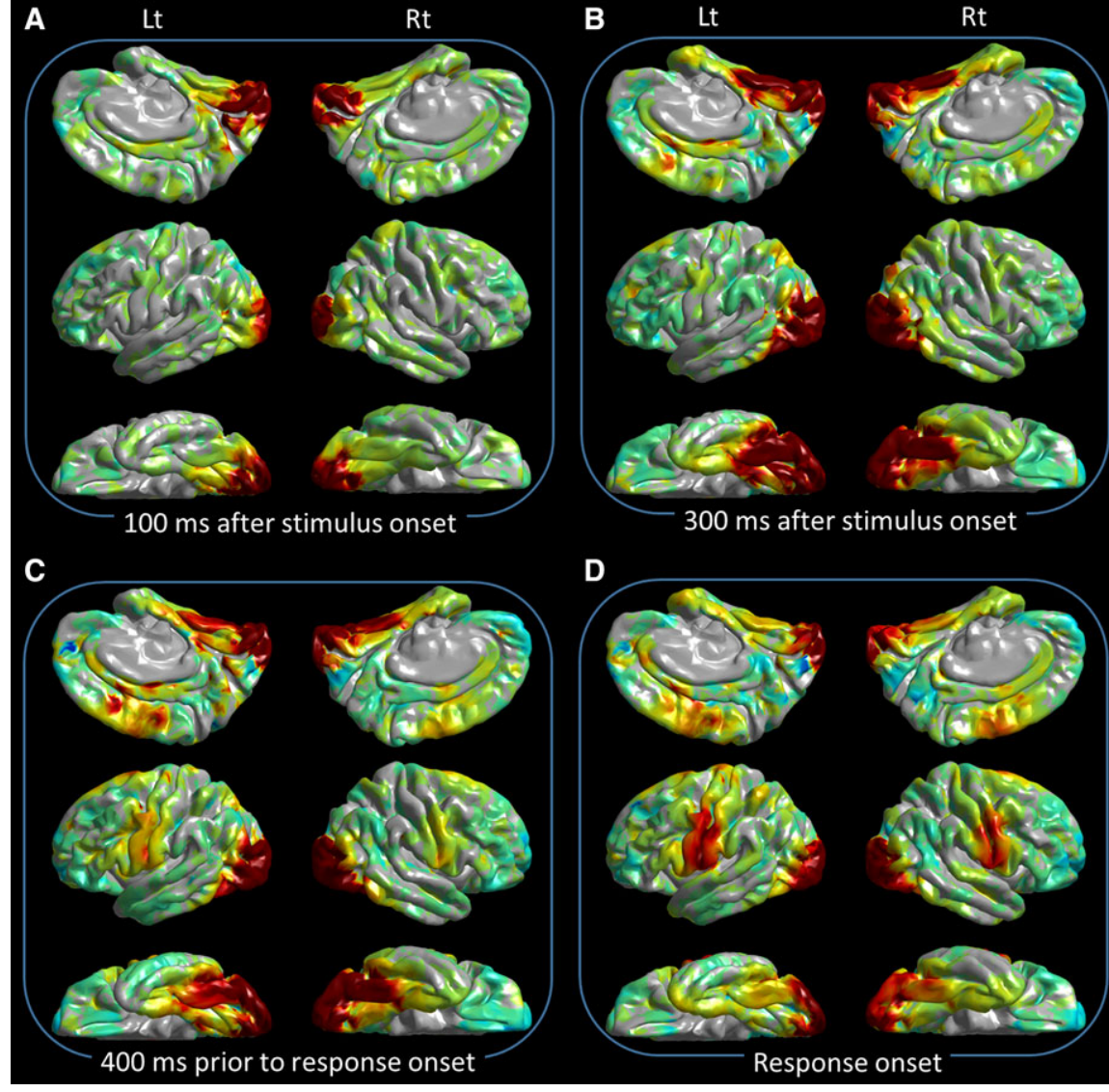

$300 \mathrm{~ms}$ after stimulus onset

D

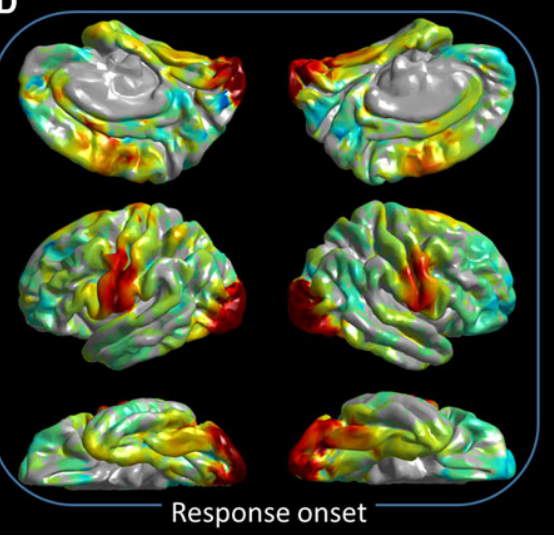

naming showed left hemisphere-specific high-gamma augmentation during this time window at 12 ROIs involving many in the left lateral prefrontal regions as well as inferior-parietal and posterior middle-temporal regions (Figure 5F-K; Video S2). Conversely, picture naming showed left-hemisphere-specific high-gamma augmentation in the pars opercularis of inferior-frontal gyrus alone. The degree of high-gamma augmentation at 328 left inferior-frontal electrode sites during this time window was much more intense in auditory compared to picture naming $(P<0.001 ;+10.6 \%$ during auditory naming vs $+0.8 \%$ during picture naming). The degree of high-gamma suppression at 304 right inferior-frontal electrode sites during this time window was not different between tasks $(P=0.28$; $-0.2 \%$ during auditory naming vs $-0.8 \%$ during auditory naming).

\subsection{Spatial-temporal characteristics of domain-common high-gamma modulation}

Commonly during picture and auditory naming tasks, the inferior-precentral regions showed high-gamma augmentation initiated at 100-300 msec following stimulus onset and sustained this until the overt response (Figure 5D).
Likewise, the inferior-postcentral regions showed highgamma augmentation initiated at $500-900 \mathrm{msec}$ before response onset (Video S2), and the posterior superior-temporal gyri showed high-gamma augmentation immediately after response onset (Figure 5C).

\section{4 | DISCUSSION}

\section{1 | Significance of domain-specific high-gamma modulation}

Our novel ECoG-based dynamic atlases revealed contrasting characteristics in the spatiotemporal profiles of namingrelated neural modulations between task domains at the whole brain level (Videos S1 and S2). Picture naming elicited early high-gamma augmentation in the occipital lobes, whereas auditory naming elicited early high-gamma augmentation in the superior-temporal gyrus over both hemispheres. High-gamma augmentation in these regions likely reflects, at least in part, lower-order perceptual processing in the visual ${ }^{25}$ or auditory domain. ${ }^{1,26}$ Previous behavioral studies have suggested that stroke in the left occipital lobe can cause difficulty in reading words or sentences partly because of the visual field deficit, with letter naming ability 


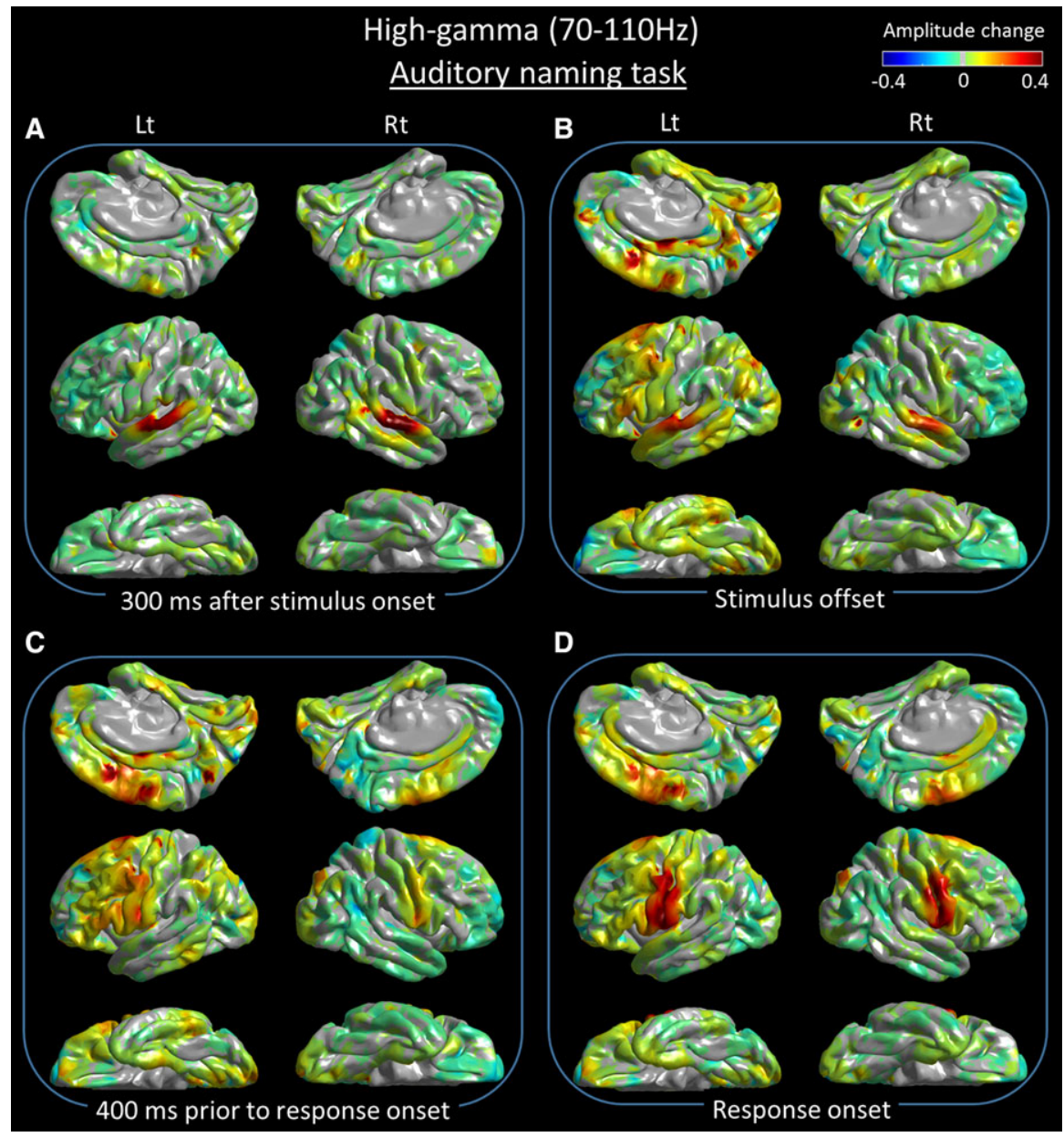

F IG URE 4 Snapshots of auditory naming-related high-gamma activity. A, $300 \mathrm{msec}$ after stimulus onset. B, Stimulus offset. C, $400 \mathrm{msec}$ before response onset. $\mathrm{D}$, Response onset being reasonably well maintained. ${ }^{27}$ The left posterior superior-temporal gyrus has been suggested to be involved in both lower-order acoustic processing and critical phonologic processing that is ultimately necessary for reproduction of speech sounds. ${ }^{28}$ Our previous study demonstrated that stimulation at the left superior-temporal gyrus resulted in either auditory hallucination or naming error. ${ }^{12}$

The left posterior temporal lobe neocortex showed a differential spatial gradient in the degree of high-gamma augmentation between task domains (Figure 5E-G). More medial structures including the fusiform gyrus showed high-gamma augmentation relatively greater during picture naming. Conversely, more lateral structures including the posterior middle-temporal gyrus showed high-gamma augmentation relatively greater during auditory naming. These observations are consistent with previous electrical stimulation, fMRI, and ECoG studies..$^{10,29,30}$ The observed spatial gradient provides a potential explanation for postoperative decline in visual more than auditory naming skills, as reported in a systematic review of 21 behavioral studies of patients undergoing left anterior-medial temporal lobe resection. ${ }^{14}$ It is plausible to speculate that extensive resection of the medial temporal lobe structures, as commonly performed in left anterior-medial temporal lobe resection, ${ }^{31}$ may be associated with collateral damage of the neural pathways that preferentially support picture naming function.

Contrasting spatiotemporal characteristics of namingrelated high-gamma augmentation noted in the left posterior temporal neocortex between tasks support the notion that the exact boundary of Wernicke's region may be difficult to define universally using the anatomic landmarks alone and that the definition has largely varied across investigators. ${ }^{32}$ Although the left posterior superior-temporal gyrus, among perisylvian structures, was most commonly treated as a part of Wernicke's region in previous reports, ${ }^{32,33}$ picture naming-related high-gamma augmentation in the left superior-temporal gyrus was minimal before response onset but became significant during response. Commonly during picture- and auditory-naming tasks, high-gamma augmentation involved the left posterior inferior-temporal gyrus before response onset (Figure 5F). Thus, our ECoG study supports the previous fMRI and lesion-deficit studies, reporting that the left posterior inferior-temporal gyrus supports semantic processing in both visual and auditory domains. ${ }^{30,34}$

The left inferior-frontal gyrus showed early high-gamma suppression subsequently followed by high-gamma 
Picture naming task

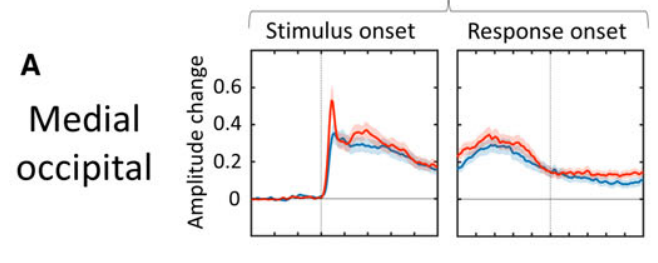

B

Lateral

occipital

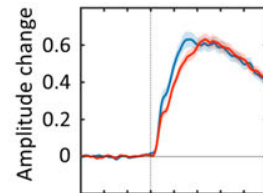

C

Posterior
superior
temporal

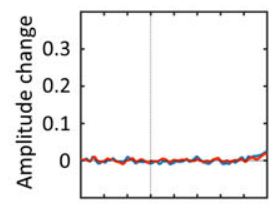

D

Inferior

precentral

Left

Right
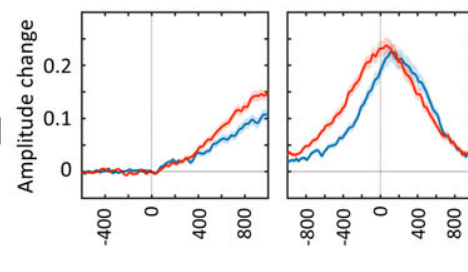

Picture naming task
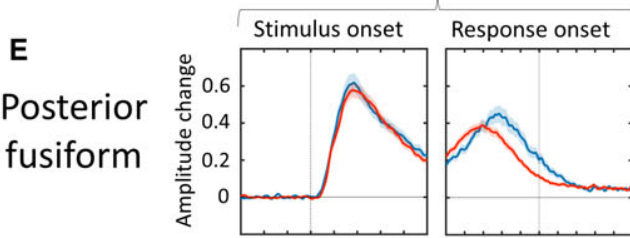

F
Posterior
inferior
temporal
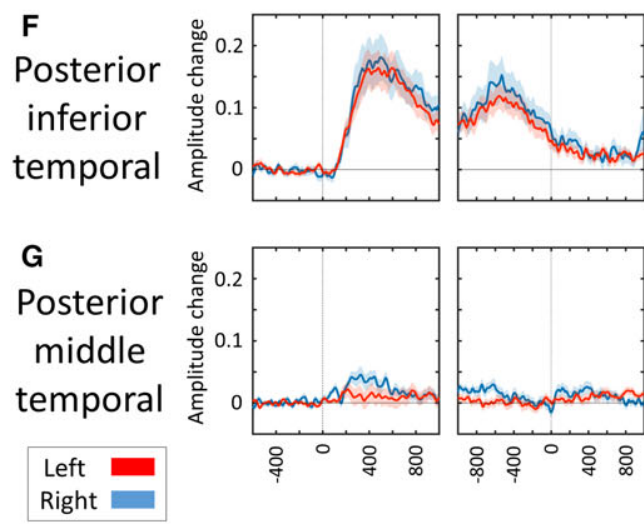

Auditory naming task
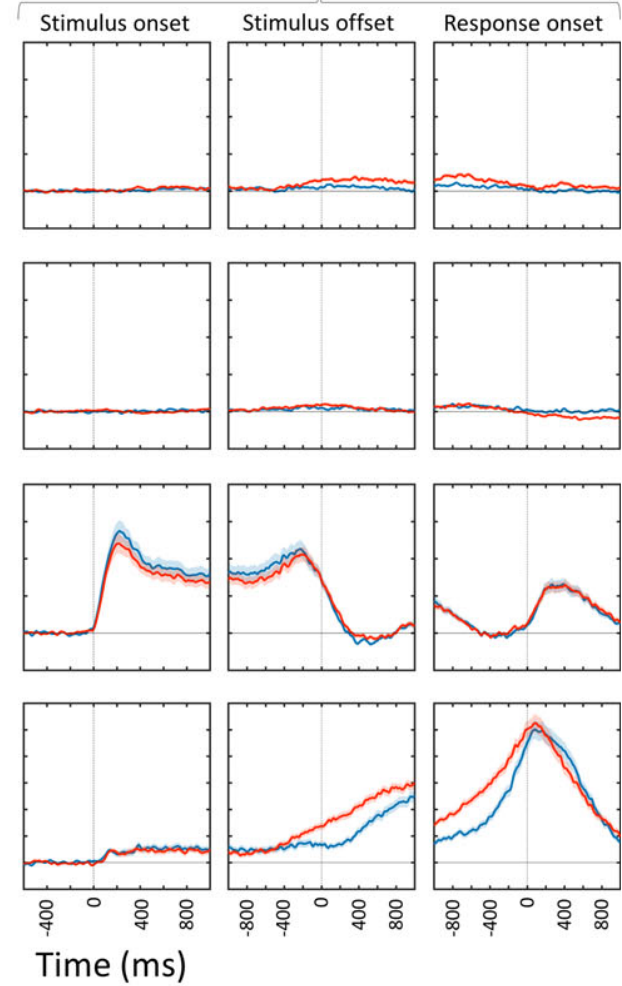

Auditory naming task
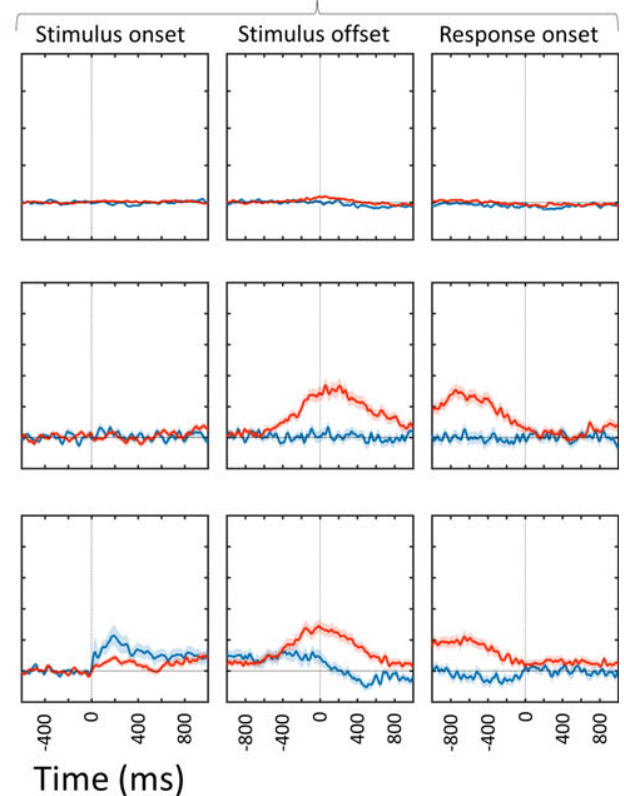

F IGURE 5 Region of interest analysis of naming-related high-gamma activity. A, Medial-occipital region. B, Lateral-occipital region. C, Posterior-superior temporal region. D, Inferior-precentral region. Video S2 shows the time-frequency plots of naming-related high-gamma activity at the whole brain level. E, Posterior fusiform region. F, Posterior inferior-temporal region. G, Posterior middle-temporal region. H, Pars opercularis. I, Pars triangularis. J, Pars orbitalis of the inferior-frontal gyrus. K, Caudal middle-frontal gyrus

augmentation before the response phase during both naming tasks. Early high-gamma suppression was more intense (picture: $-2.9 \%$ vs auditory: $-1.3 \%$ ) and subsequent augmentation less intense $(+0.8 \%$ vs $+10.6 \%)$ in picture naming as compared to auditory naming. The results of our study are consistent with those of previous studies, reporting that hemodynamic and neural activation in the left frontal lobe was greater during auditory naming compared 


\section{Picture naming task}

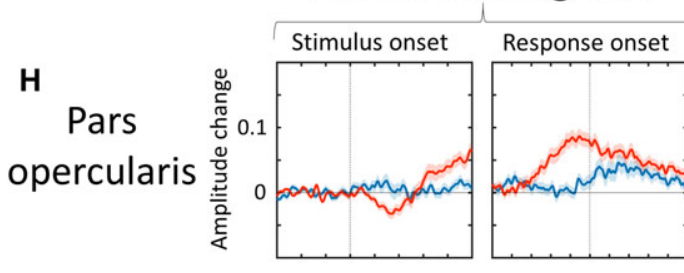

I triangularis
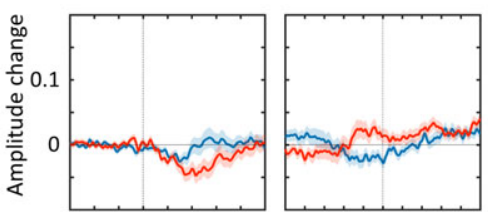

J

Pars orbitalis
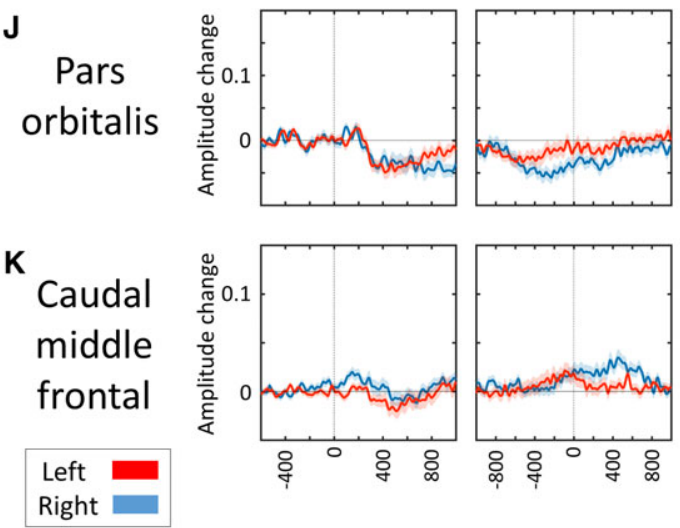

Auditory naming task
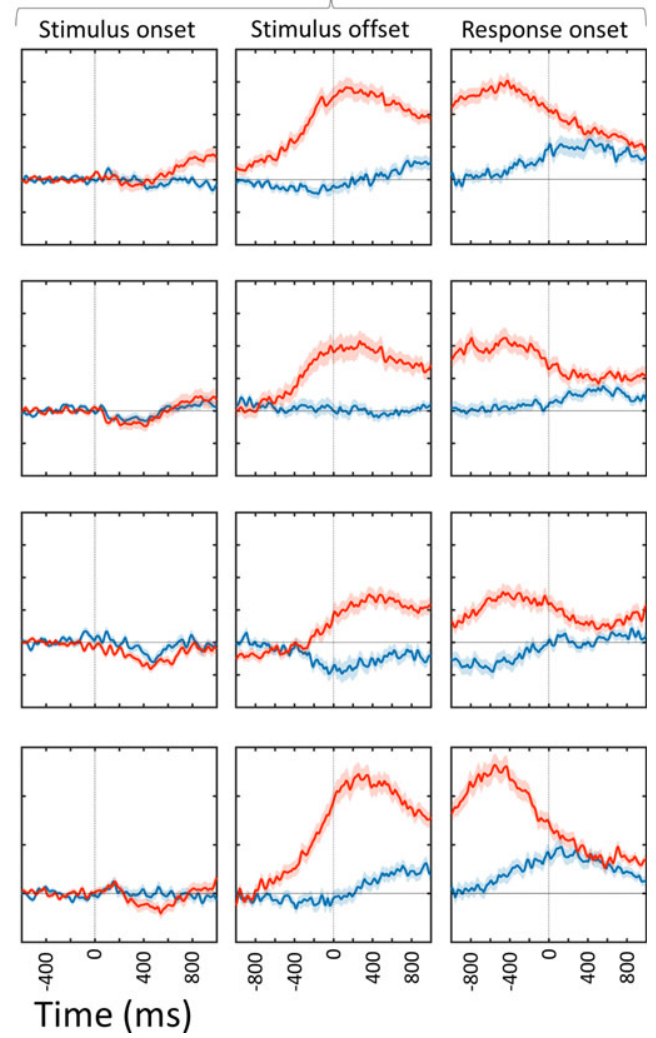

FIGURE 5 Continued

to during picture naming. ${ }^{10,30}$ Collective evidence indicates that the picture-naming task can be completed with less activation of the left lateral prefrontal regions including the left inferior-frontal gyrus, known to include Broca's area. Less-extensive activation of the left inferior-frontal gyrus during picture naming can be attributed in part to the experimental paradigms employed. Picture naming does not require syntactic processing as required by auditory naming, and the left inferior-frontal gyrus is suggested to support syntactic function. ${ }^{35,36}$ Recent ECoG studies showed that inferior-frontal high-gamma augmentation was fairly symmetric during an auditory verbal working memory task, which requires neither syntactic nor semantic processing, ${ }^{37}$ but highly left-hemispheric dominant in an auditory descriptive naming task ${ }^{12}$ such as the one employed herein. Other cognitive processes might also account for a greater extent of high-gamma augmentation in the left lateral prefrontal regions during auditory naming. A previous ECoG study reported that high-gamma augmentation within the left lateral prefrontal regions including the inferior-frontal gyrus was more intense during naming of ambiguous images compared to naming of unambiguous common objects. $^{38}$ The former naming task also requires "estimation" and "selection," whereas the latter naming task can be completed rapidly in an automatic manner. ${ }^{38}$
The left inferior precentral gyrus commonly showed highgamma augmentation within 100-300 msec following stimulus onset, earliest among all frontal lobe ROIs (Figure 5). In the same time window, high-gamma activity was rather suppressed in the left inferior-frontal gyrus during picture- and auditorynaming tasks. These observations do not support the classic language model, as illustrated in the previous research, ${ }^{32,39}$ that internal word image generated in the left temporoparietal neocortex is transferred initially and solely to the left inferior-frontal gyrus and subsequently to the left inferior precentral gyrus in a rostral-to-caudal direction. Based on the theory of antagonism, ${ }^{16,17}$ one can hypothesize that early suppression of the unnecessary function in the left inferior-frontal gyrus may facilitate effective completion of the naming task or that energy resources may be distributed optimally to the regions actively processing stimuli. Our recent ECoG study utilizing an auditory verbal working memory task suggested that the left inferior-precentral gyrus supports initial maintenance of a mental representation of memory items, whereas the left inferior-frontal gyrus contributes to later scanning function to determine a match among previously encountered items. ${ }^{37}$ Studies utilizing corticocortical evoked potentials and diffusion tensor tractography have demonstrated that the left inferior-precentral gyrus is effectively connected structurally and functionally to both the superior-temporal and inferior-frontal gyri. ${ }^{40,41}$ 


\section{2 $\mid$ Methodologic considerations}

We cannot rule out the possibility that the epileptogenic zone may have affected the spatiotemporal dynamics of naming-related high-gamma activity. Indeed, we reported previously that $\mathrm{SOZ}$, compared to non-SOZ, has a smaller chance of generating significant naming-related highgamma augmentation. ${ }^{11}$ Thus, we have excluded both SOZ and sites affected by a structural lesion from further analysis. Our observation of high-gamma augmentation that is spatially different across auditory and visual domains cannot be explained by the effect of SOZ localization, because ECoG signals between tasks were compared within the same patient cohort. Furthermore, interictal spike discharges per se are accompanied by a pathologic increase in highgamma power. ${ }^{42,43}$ Thus, the present study also excluded electrode sites showing interictal epileptiform discharges during the task, as it is a common practice in ECoG-based cognitive neuroscience research. ${ }^{3}$ Our previous ECoG study of 4 patients with left frontal lobe epilepsy reported that slow waves immediately following interictal spikes may transiently inhibit naming-related cortical activity. However, this appeared to be restricted to regions involved in spikeand-wave discharge and did not appear to affect sites not involved in spike-and-wave discharges. ${ }^{44}$ Further analysis of a large number of patients at the whole brain level is necessary to better determine the transient effects of focal spike-and-wave discharges on the language network, taking into account that interictal focal spike discharges are suggested to have an impact on widespread networks beyond the regions from which spikes are generated. ${ }^{45}$

Subdural disk electrodes used in the present study do not effectively sample ECoG signals from the cortex facing a sulcus. Further studies using depth electrodes may be warranted to determine if high-gamma augmentation in the superior-temporal gyri is attributed to activation of the immediately underlying cortex or that in the planum temporale.

Our patient cohort included those of age ranging from 5 to 44 years. Thus, great care needs to be taken in interpreting the results of time-frequency analysis. The spatial-temporal dynamics of averaged naming-related high-gamma activity, as presented in Video S1, likely reflect the neural dynamics exerted commonly by patients across a wide range of ages. In other words, neural dynamics exerted by a specific age group alone may have been underestimated. Development of the language systems is expected to be incomplete in younger children. ${ }^{46}$ Our previous ECoG studies of auditory naming-related neural dynamics reported that older age was associated with a greater degree of inferior-precentral high-gamma augmentation during stimulus listening as well as a greater degree of left rostral middle-frontal high-gamma augmentation immediately following stimulus offset. ${ }^{12,47}$ In the present study, younger patients had a longer response time compared to older ones. The effect of variance in response time across age is expected to be more prominent in the time windows long after stimulus onset as well as long before response onset. To distinguish high-gamma modulations related to perceptual and motor processing, we have provided the time-frequency plots that are time-locked to stimulus onset and response onset, respectively.

We employed a multivariate linear regression analysis to determine whether the response accuracy was independently associated with age, age at epilepsy onset, history of generalized tonic-clonic seizures (GTCS), or each of the 8 lobes affected by seizure-onset zone (ie, left frontal, left temporal, left parietal, left occipital, right frontal, right temporal, right parietal, and right occipital lobes; Table 1). This post hoc analysis failed to demonstrate the association between the response accuracy and any of the aforementioned 11 variables during either naming task (uncorrected $P>0.05$ ).

\section{3 | Future directions}

Although the present study demonstrated that auditory naming, compared to picture naming, elicited more extensive high-gamma augmentation, which involved the left lateral prefrontal and posterior cingulate regions, we still do not know whether auditory naming is more useful than picture naming for localization of the critical language areas or prediction of language outcome following cortical resection. Correlation between naming-related high-gamma activity and postoperative neuropsychological assessment in large populations will be ultimately necessary to address this question. A recent systematic review infers that the extent of cortex showing auditory or picture naming-related high-gamma augmentation was more extensive than the critical language area defined by electrical stimulation mapping in pediatric populations. ${ }^{9}$ Conversely, resection of naming-related high-gamma sites can result in postoperative language impairment, even though stimulation-defined critical language areas were preserved. ${ }^{10,11}$

The strength of ECoG-based language mapping includes its more robust signal fidelity against overt speech-related artifacts and its capability to determine the detailed temporal dynamics of task-related neural modulations. Thus, inclusion of larger numbers of patients with a wide range of age in future collaborative works is expected to determine the effect of age on naming-related high-gamma modulations at given time windows at the whole brain level. We plan to test our hypothesis that efficient naming is supported by selective elaboration of new network connectivity throughout development. We are willing to share our dataset with investigators who express an interest in studying the neurobiology of language or the clinical utility of naming-related high-gamma activity. 


\section{ACKNOWLEDGMENTS}

This work was supported by National Institutes of Health (NIH) grants NS047550, NS064033, MH107512, and NS089659. We are grateful to Alanna Carlson, MS, LLP, Karin Halsey, BS, REEGT, and Jamie MacDougall RN, BSN, CPN, at Children's Hospital of Michigan, Detroit Medical Center, Wayne State University, for research coordination and assistance in data acquisition.

\section{DISCLOSURE}

None of the authors has any conflict of interest to disclose. We confirm that we have read the Journal's position on issue involved in ethical publication and affirm that this report is consistent with those guidelines.

\section{ORCID}

Eishi Asano (D) https://orcid.org/0000-0001-8391-4067

\section{REFERENCES}

1. Arya R, Wilson JA, Fujiwara $\mathrm{H}$, et al. Electrocorticographic high-gamma modulation with passive listening paradigm for pediatric extraoperative language mapping. Epilepsia. 2018;59:792-801.

2. Ojemann G, Ojemann J, Lettich E, et al. Cortical language localization in left, dominant hemisphere. An electrical stimulation mapping investigation in 117 patients. J Neurosurg. 1989;71:31626.

3. Crone NE, Sinai A, Korzeniewska A. High-frequency gamma oscillations and human brain mapping with electrocorticography. Prog Brain Res. 2006;159:275-95.

4. Austermuehle A, Cocjin J, Reynolds R, et al. Language functional MRI and direct cortical stimulation in epilepsy preoperative planning. Ann Neurol. 2017;81:526-37.

5. Ball T, Kern M, Mutschler I, et al. Signal quality of simultaneously recorded invasive and non-invasive EEG. NeuroImage. 2009;46:708-16.

6. Ray S, Crone NE, Niebur E, et al. Neural correlates of highgamma oscillations $(60-200 \mathrm{~Hz})$ in macaque local field potentials and their potential implications in electrocorticography. J Neurosci. 2008;28:11526-36.

7. Scheeringa R, Fries P, Petersson KM, et al. Neuronal dynamics underlying high- and low-frequency EEG oscillations contribute independently to the human BOLD signal. Neuron. 2011;69:572-83.

8. Nishida M, Juhász C, Sood S, et al. Cortical glucose metabolism positively correlates with gamma-oscillations in nonlesional focal epilepsy. NeuroImage. 2008;42:1275-84.

9. Arya R, Horn PS, Crone NE. ECoG high-gamma modulation versus electrical stimulation for presurgical language mapping. Epilepsy Behav. 2018;79:26-33.

10. Cervenka MC, Corines J, Boatman-Reich DF, et al. Electrocorticographic functional mapping identifies human cortex critical for auditory and visual naming. NeuroImage. 2013;69:267-76.
11. Kojima K, Brown EC, Rothermel R, et al. Clinical significance and developmental changes of auditory-language-related gamma activity. Clin Neurophysiol. 2013;124:857-69.

12. Nakai Y, Jeong JW, Brown EC, et al. Three- and four-dimensional mapping of speech and language in patients with epilepsy. Brain. 2017;140:1351-70.

13. Fukuda M, Juhász C, Hoechstetter K, et al. Somatosensory-related gamma-, beta- and alpha-augmentation precedes alpha- and betaattenuation in humans. Clin Neurophysiol. 2010;121:366-75.

14. Ives-Deliperi VL, Butler JT. Naming outcomes of anterior temporal lobectomy in epilepsy patients: a systematic review of the literature. Epilepsy Behav. 2012;24:194-8.

15. Kojima K, Brown EC, Matsuzaki N, et al. Gamma activity modulated by picture and auditory naming tasks: intracranial recording in patients with focal epilepsy. Clin Neurophysiol. 2013; 124:1737-44.

16. Fox MD, Snyder AZ, Vincent JL, et al. The human brain is intrinsically organized into dynamic, anticorrelated functional networks. Proc Natl Acad Sci USA. 2005;102:9673-8.

17. Anticevic A, Cole MW, Murray JD, et al. The role of default network deactivation in cognition and disease. Trends Cogn Sci. 2012;16:584-92.

18. Kambara T, Sood S, Alqatan Z, et al. Presurgical language mapping using event-related high-gamma activity: the Detroit procedure. Clin Neurophysiol. 2018;129:145-54.

19. Asano E, Benedek K, Shah A, et al. Is intraoperative electrocorticography reliable in children with intractable neocortical epilepsy? Epilepsia. 2004;45:1091-9.

20. Asano E, Juhász C, Shah A, et al. Role of subdural electrocorticography in prediction of long-term seizure outcome in epilepsy surgery. Brain. 2009;132:1038-47.

21. Desikan RS, Ségonne F, Fischl B, et al. An automated labeling system for subdividing the human cerebral cortex on MRI scans into gyral based regions of interest. NeuroImage. 2006;31:968-80.

22. Rossion B, Pourtois G. Revisiting Snodgrass and Vanderwart's object pictorial set: the role of surface detail in basic-level object recognition. Perception. 2004;33:217-36.

23. Papp N, Ktonas P. Critical evaluation of complex demodulation techniques for the quantification of bioelectrical activity. Biomed Sci Instrum. 1977;13:135-45.

24. Hoechstetter K, Bornfleth H, Weckesser D, et al. BESA source coherence: a new method to study cortical oscillatory coupling. Brain Topogr. 2004;16:233-8.

25. Nakai Y, Nagashima A, Hayakawa A, et al. Four-dimensional map of the human early visual system. Clin Neurophysiol. 2018;129:188-97.

26. Mooij AH, Huiskamp GJM, Gosselaar PH, et al. Electrocorticographic language mapping with a listening task consisting of alternating speech and music phrases. Clin Neurophysiol. 2016;127:1113-9.

27. Leff AP, Crewes H, Plant GT, et al. The functional anatomy of single-word reading in patients with hemianopic and pure alexia. Brain. 2001;124:510-21.

28. Hickok G, Poeppel D. The cortical organization of speech processing. Nat Rev Neurosci. 2007;8:393-402.

29. Hamberger MJ, McClelland S 3rd, McKhann GM 2nd, et al. Distribution of auditory and visual naming sites in nonlesional temporal lobe epilepsy patients and patients with space-occupying temporal lobe lesions. Epilepsia. 2007;48:531-8. 
30. Hamberger MJ, Habeck CG, Pantazatos SP, et al. Shared space, separate processes: neural activation patterns for auditory description and visual object naming in healthy adults. Hum Brain Mapp. 2014;35:2507-20.

31. Spencer DD, Spencer SS, Mattson RH, et al. Access to the posterior medial temporal lobe structures in the surgical treatment of temporal lobe epilepsy. Neurosurgery. 1984;15:667-71.

32. Tremblay P, Dick AS. Broca and Wernicke are dead, or moving past the classic model of language neurobiology. Brain Lang. 2016;162:60-71.

33. Bogen JE, Bogen GM. Wernicke's region - where is it? Ann NY Acad Sci. 1976;280:834-43.

34. DeLeon J, Gottesman RF, Kleinman JT, et al. Neural regions essential for distinct cognitive processes underlying picture naming. Brain. 2007;130:1408-22.

35. Dapretto M, Bookheimer SY. Form and content: dissociating syntax and semantics in sentence comprehension. Neuron. 1999;24:427-32.

36. Hagoort P. On Broca, brain, and binding: a new framework. Trends Cogn Sci. 2005;9:416-23.

37. Kambara T, Brown EC, Jeong JW, et al. Spatio-temporal dynamics of working memory maintenance and scanning of verbal information. Clin Neurophysiol. 2017;128:882-91.

38. Cho-Hisamoto Y, Kojima K, Brown EC, et al. Gamma activity modulated by naming of ambiguous and unambiguous images: intracranial recording. Clin Neurophysiol. 2015;126:17-26.

39. Tyler LK, Marslen-Wilson W. Fronto-temporal brain systems supporting spoken language comprehension. Philos Trans R Soc Lond B Biol Sci. 2008;363:1037-54.

40. Brown EC, Jeong JW, Muzik O, et al. Evaluating the arcuate fasciculus with combined diffusion-weighted MRI tractography and electrocorticography. Hum Brain Mapp. 2014;35:2333-47.

41. Nishida M, Korzeniewska A, Crone NE, et al. Brain network dynamics in the human articulatory loop. Clin Neurophysiol. 2017;128:1473-87.
42. Jacobs J, Kobayashi K, Gotman J. High-frequency changes during interictal spikes detected by time-frequency analysis. Clin Neurophysiol. 2011;122:32-42.

43. Zijlmans M, Worrell GA, Dümpelmann M, et al. How to record high-frequency oscillations in epilepsy: a practical guideline. Epilepsia. 2017;58:1305-15.

44. Brown EC, Matsuzaki N, Asano E. The transient effect of interictal spikes from a frontal focus on language-related gamma activity. Epilepsy Behav. 2012;24:497-502.

45. Fahoum F, Lopes R, Pittau F, et al. Widespread epileptic networks in focal epilepsies: EEG-fMRI study. Epilepsia. 2012;53:1618-27.

46. Weiss-Croft LJ, Baldeweg T. Maturation of language networks in children: a systematic review of 22 years of functional MRI. NeuroImage. 2015;123:269-81.

47. Kambara T, Brown EC, Silverstein BH, et al. Neural dynamics of verbal working memory in auditory description naming. Sci Rep. 2018;8:15868.

\section{SUPPORTING INFORMATION}

Additional supporting information may be found online in the Supporting Information section at the end of the article.

How to cite this article: Nakai Y, Sugiura A, Brown EC, et al. Four-dimensional functional cortical maps of visual and auditory language: Intracranial recording. Epilepsia. 2019;60:255-267. https://doi.org/10.1111/epi.14648 